\title{
Cerebral Angiography Induced Prolonged Focal Seizure and Hemiparesis during Carotid Artery Balloon Test Occlusion
}

\author{
Yoriko Kato $^{1,2 *}$, Takatoshi Sorimach', Yasushi Ito', Yukihiko Fujiii', Nobutaka Kawahara ${ }^{2}$ \\ ${ }^{1}$ Department of Neurosurgery, Brain Research Institute, University of Niigata, Niigata, Japan \\ ${ }^{2}$ Department of Neurosurgery, Yokohama City University, Yokohama, Japan \\ Email: ${ }^{*}$ yoriko@pc4.so-net.ne.jp
}

Received 27 March 2014; revised 28 April 2014; accepted 28 May 2014

Copyright $(02014$ by authors and Scientific Research Publishing Inc.

This work is licensed under the Creative Commons Attribution International License (CC BY). http://creativecommons.org/licenses/by/4.0/

(c) (9) Open Access

\section{Abstract}

The frequency of neurologic complications of cerebral angiography is $0.3 \%-2.3 \%$, and the frequency of the persistent neurologic deficits that remain over $7-10$ days is $0.4 \%-0.5 \%$. On the other hand, all neurologic complications of balloon test occlusion (BTO) have been reported in $0 \%$ $-8.3 \%$ and especially in $1.6 \%-1.7 \%$ of symptomatic neurologic complications. There are a few reports concerning a seizure related to cerebral angiography and BTO. We report a case of prolonged seizures and left hemiparesis during and after BTO of the right internal carotid artery. The patient showed repeated focal seizures and prolonged left hemiparesis lasting for approximately 2 months. Computed tomography and magnetic resonance imaging demonstrated no abnormal findings. Suspected mechanisms of seizure were a cerebral blood flow change induced by BTO and neurotoxicity of accumulated contrast medium. A combination of prolonged focal seizures and subsequent hemiparesis is a rare complication of BTO, which should be taken into consideration during BTO.

\section{Keywords}

Balloon Test Occlusion, Cerebral Angiography, Hemiparesis, Seizure

\section{Introduction}

Cerebral angiography has been reported to cause persisting neurologic complications in $0.4 \%-0.5 \%$ of the procedures [1] [2]. The reported rate of symptomatic neurologic complication related to BTO has been $1.6 \%-1.7 \%$

\footnotetext{
"Corresponding author.
}

How to cite this paper: Kato, Y., Sorimach, T., Ito, Y., Fujii, Y. and Kawahara, N. (2014) Cerebral Angiography Induced Prolonged Focal Seizure and Hemiparesis during Carotid Artery Balloon Test Occlusion. Open Journal of Modern Neurosurgery, 4, 126-130. http://dx.doi.org/10.4236/ojmn.2014.43022 
[3] [4]. Few studies reported seizures induced by cerebral angiography and/or BTO [5] [6]. We report a rare case of repeated focal seizures and prolonged left hemiparesis after cerebral angiography and BTO of the right internal carotid artery.

\section{Case Report}

A 51-year-old female with an unruptured aneurysm at the junction of the right internal carotid artery (ICA) and the ophthalmic artery was admitted to our hospital for a preoperative evaluation. She had a history of seizures with fever in childhood and again at 18 years old. We conducted a preoperative angiography and a BTO of the right ICA to evaluate collateral flows. Before the angiography, a bolus heparin of 5000 units was administered intravenously and the activated clotting time reached 282 sec. We administered ioxaglic acid (Hexabrix, Guerbet Japan, Tokyo, Japan) at a rate of $3 \mathrm{~mL} / \mathrm{s}$ and total amount of $5 \mathrm{~mL}$ for the right ICA angiography (Figure 1(a), Figure 1(b)). Three-dimensional rotation angiography of the right ICA was performed. Subsequently, a Selecon MP balloon catheter of 5.2 Fr. (Terumo Clinical Supply, Gifu, Japan) was placed at just distal of the right ICA's orifice. The balloon was inflated and a contrast medium was injected manually to confirm occlusion of the right ICA (Figure 1(c)). Immediately after confirming the stagnation of the contrast medium in the ICA on fluoroscopy, the patient became unconsciousness with conjugate deviation of bilateral eyeballs to the left side and with tetraparesis. The balloon catheter was deflated promptly. The right ICA angiogram performed soon after the balloon deflation showed no abnormal findings of the arteries in the right carotid artery territory. Intravenous injection of $15 \mathrm{mg}$ of diazepam and $500 \mathrm{mg}$ of phenytoin were administered. CT performed just after the procedure revealed no abnormal findings. Phenobarbital of $100 \mathrm{mg}$ a day and predonisolone of $40 \mathrm{mg}$ a day were administered with sufficient intravenous hydration. For the 12 hours after the procedure, she was unconscious with clonic movement of her left limbs. MRI and MRA performed the day after the procedure showed no abnormal findings (Figure 2). For 5 days after the procedure, intermittent transient consciousness disturbance with the conjugate deviation of her eyes to the left side and clonic involuntary movement of her left limbs occurred 2 - 3 times a day. Left hemiparesis was found during the interval of the seizures. Five days after the procedure, oral administration of carbamazepine of $200 \mathrm{mg}$ a day was started and increased to $700 \mathrm{mg}$ a day with monitoring of the blood concentration. Her epileptic fits disappeared 8 days after the procedure. An electroencephalography performed during the intermittent period of the attack showed slow waves in the right frontal with no apparent spike wave (Figure 3). MRIs performed 8 and 21 days after the procedure showed no abnormality. Carbamazepine was changed to levetiracetam of $500 \mathrm{mg}$ a day, because of drug eruption caused by the carbamazepine. The transient clonic movement of her left limbs recurred 33 days after the procedure and then the ictus occurred

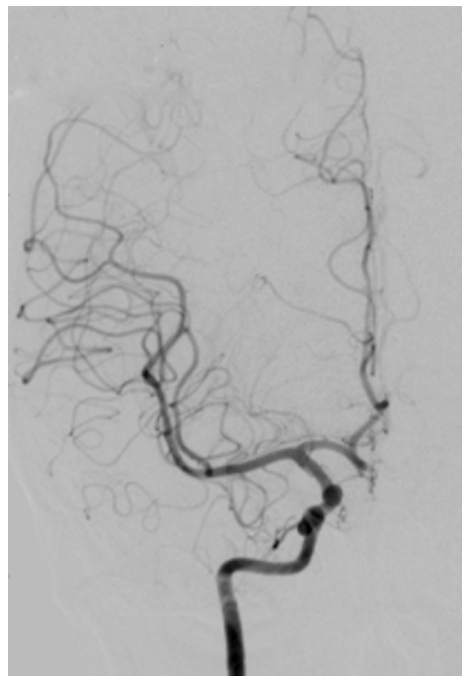

(a)

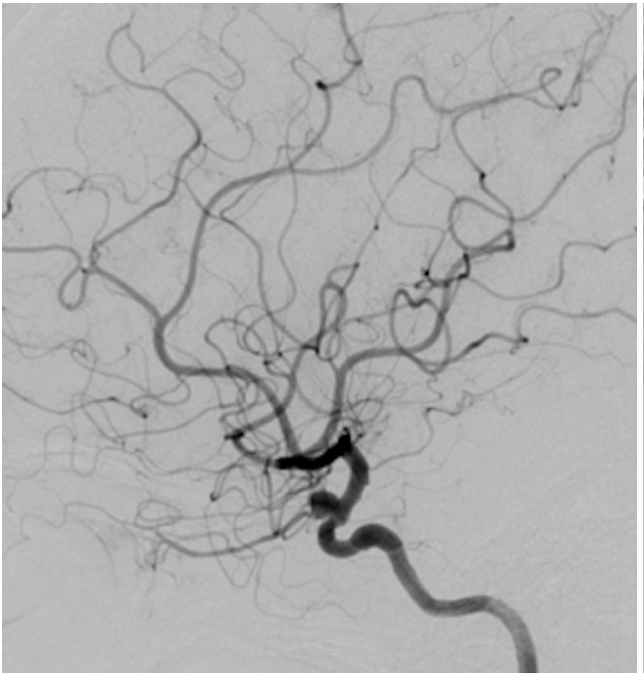

(b)

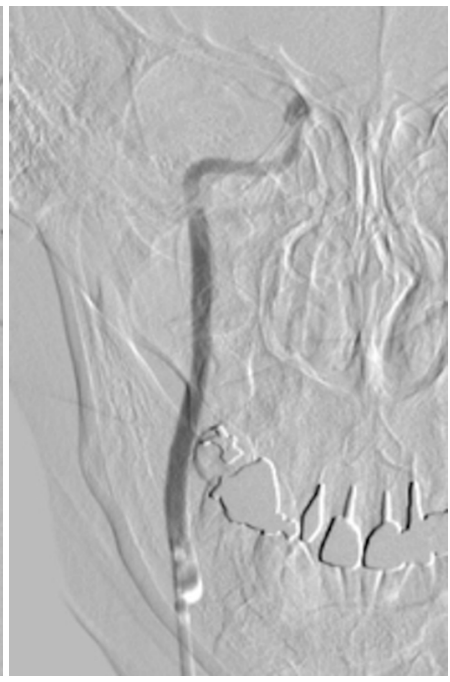

(c)

Figure 1. A right internal carotid angiogram showing an internal carotid-ophthalmic artery aneurysm in anteroposterior view (a) and in lateral view (b). (c) A right internal carotid angiogram during balloon occlusion of the right internal carotid artery showing stagnant contrast medium in the internal carotid artery. 


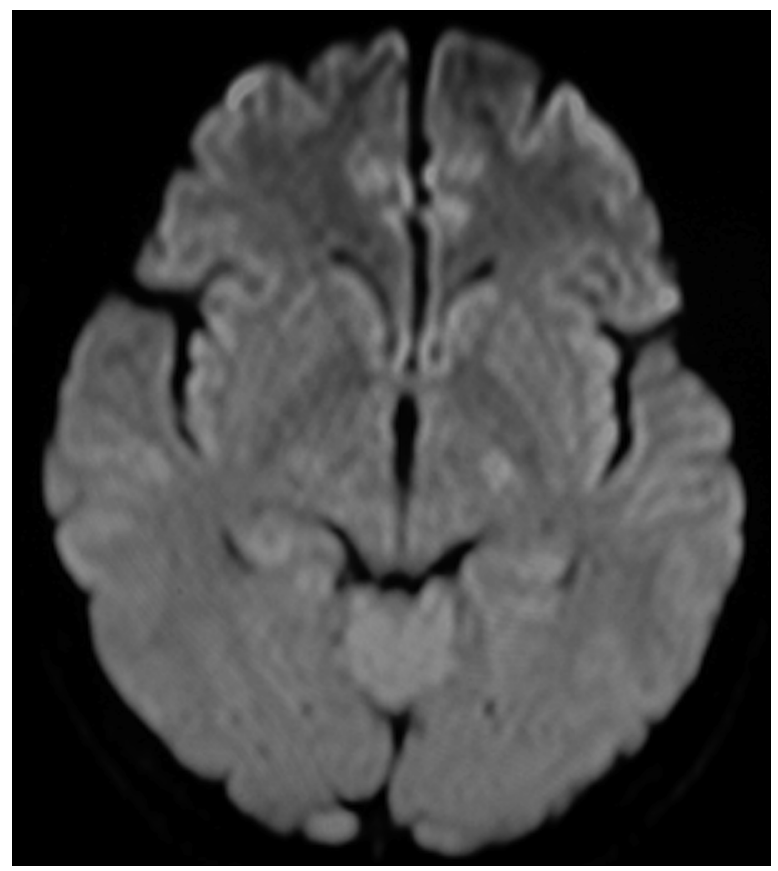

Figure 2. A diffusion-weighted MRI performed the day after balloon test occlusion reveals no ischemic lesion.

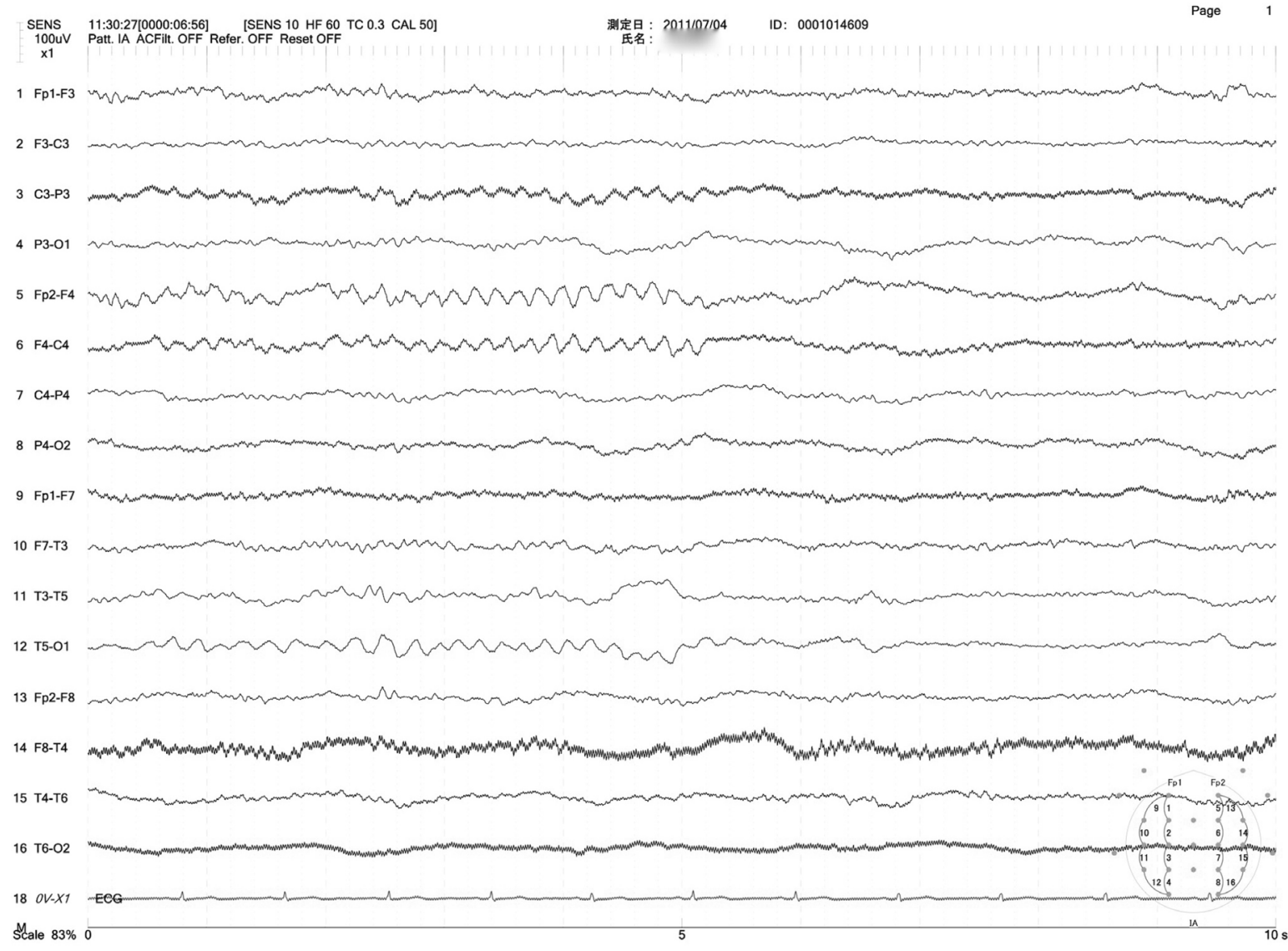

Figure 3. An electroencephalogram obtained 5 days after balloon test occlusion showing slow waves in the right frontal area. 
every day. Because secondary generalization following the clonic movement occurred, valproic acid was added. Levetiracetam and valproic acid were increased to $1500 \mathrm{mg}$ and $400 \mathrm{mg}$ a day, respectively, finally resulting in the disappearance of the attacks. The patient was discharged 60 days after the procedure with slight left hemiparesis.

\section{Discussion}

Among neurologic complications caused by cerebral angiography, few cases of seizures induced by contrast media have been reported [5] [6]. Mitsuyama et al. reported intractable focal seizures induced by cerebral angiography using iomeprol in a patient with venous angioma in the left cerebellum. In the report, tonic movement of the left arm occurred during the right carotid angiography and it continued intermittently for approximately 2 hours, followed by left arm weakness persisting for 60 hours after the angiography. Because no abnormality was demonstrated on CT and MRI, they speculated that mechanisms of the neurologic complication were disruption of the blood brain barrier (BBB) by the contrast medium and it's neurotoxicity to the brain [5].

Main reported causes of neurologic complications induced by BTO were carotid artery dissections and arterial embolism [3] [4]. Haraguchi et al. has reported seizures caused by BTO. In the report, a patient with a right mandibular ameloblastoma developed left hemiparesis followed by a generalized seizure, which resolved completely 4 days after the procedure. T2-weighted MRI and diffusion-weighted MRI revealed an abnormal hyperintense lesion in the right frontal lobe. They speculated that a possible mechanism of the seizure was vasogenic edema induced by the temporary ischemia and reperfusion of the BTO [7]. During endovascular treatment with transient arterial occlusion using a balloon catheter, occurrence of cerebral enhancement by contrast medium has been reported, which was possibly induced by reperfusion injury leading to BBB disruption [8]. To the best of our knowledge, hemiparesis persisting for longer than 2 months following seizures induced by BTO has never been reported previously.

Ioxaglic acid has proved to be the most anticoagulant contrast agent as a result of a combination of its osmolality and its ionic strength. We usually use Ioxaglic acid during cerebral angiography in consideration for this characteristic. Non-ionic compounds bring benefit and fewer adverse side effects associated with their reduced chemotoxicity and high hydrophilicity, using a non-ionic contrast agent might have prevented the neurological complications in our case [9].

Nelson et al. reported that 22 of 29 patients (76\%) with contrast medium-induced seizures had a history of previous spontaneous seizures in their consecutive series of contrast-enhanced head CT examinations [10]. Considering the seizure history of our patient, the use of a non-ionic contrast medium and/or advance administration of anti-epileptics might have prevented the neurological complications. In case of convulsions induced by contrast media, sufficient intravenous hydration and early administration of carbamazepine with monitoring of its blood concentration are recommended.

\section{Conclusion}

We report a unique case that manifested with a prolonged seizure and hemiparesis during BTO. Because angiography performed immediately after undergoing BTO and MRI didn't reveal embolic event, we speculated that neurotoxicity of ionic contrast medium induced neurologic complications. During the BTO procedure, transient ischemia, reperfusion, and the attenuation of the contrast agent may influence the neurotoxicity of contrast agent. In patients with a history of seizures, it is necessary to pay attention to incomplete continuous cerebral ischemia caused by BTO and balloon-assist techniques, which may induce seizures accompanied with hemiparesis.

\section{References}

[1] Leffers, A.M. and Wagner, A. (2000) Neurologic Complications of Cerebral Angiography. Acta Radiologica, 41, 204210. http://dx.doi.org/10.1080/028418500127345299

[2] Willinsky. R.A., Taylor, S.M., TerBrugge. K., Farb, R.I., Tomlinson, G. and Montanera, W. (2003) Neurologic Complications of Cerebral Angiography: Prospective Analysis of 2,899 Procedures and Review of the Literature. Neuroradiology, 22, 522-528.

[3] Mathis, J.M., Barr, J.D., Jungreis, C.A., Yonas, H., Sekhar, L.N., Vincent, D., Pentheny, S.L. and Horton, J.A. (1995) Temporary Balloon Test Occlusion of the Internal Carotid Artery: Experience in 500 Cases. American Journal of Neuroradiology, 16, 749-754. 
[4] Tarr, R.W., Jungreis, C.A., Horton, J.A., Petheny, S., Sekhar, L.N., Sen, C., Janecka, I.P. and Yonas, H. (1991) Complications of Preoperative Balloon Test Occlusion of the Internal Carotid Arteries: Experience in 300 Cases. Skull Base Surgery, 1, 240-244. http://dx.doi.org/10.1055/s-2008-1057104

[5] Mitsuyama, T., Sato, S., Ishii, A. and Kawamata, T. (2010) Contrast Medium-Induced Seizures and Prolonged Motor Weakness after Cerebral Angiography: Case Report. Neurosurgery, 67, 1460-1463. http://dx.doi.org/10.1227/NEU.0b013e3181f352a1

[6] Mutoh, T., Ishikawa, T., Sawada, M., Moroi, J., Tamakawa, N., Hikichi, K., Suzuki, A. and Yasui, N. (2010) Cerebral Complications Induced by Neurotoxity of Nonionic Contrast Medium after Embolization of Unruptured Cerebral Aneurysms: Report of 2 Cases. No Shinkei Geka, 38, 163-170.

[7] Haraguchi, K., Houkin, K., Koyanagi, I., Nonaka, T. and Baba, T. (2009) Reversible Hyperintense Lesion after Balloon Occlusion Test. Journal of Neuroimaging, 19, 174-178. http://dx.doi.org/10.1111/j.1552-6569.2007.00223.x

[8] Ozturk, A., Saatci, I., Pamuk, A.G., Erdogan, C., Akmangit, I., Geyik, S. and Cekirge, H.S. (2006) Focal Increased Cortical Density in Immediate Postembolization CT Scans of Patients with Intracranial Aneurysms. American Journal of Neuroradiology, 27, 1866-1875.

[9] McClennan, B.L. (1990) Ionic and Nonionic Iodinated Contrast Media: Evolution and Strategies for Use. American Journal of Roentgenology, 155, 225-233. http://dx.doi.org/10.2214/ajr.155.2.2115244

[10] Nelson, M., Bartlett, R.J. and Lamb, J.T. (1989) Seizure after Intravenous Contrast Media for Cranial Computed Tomography. Journal of Neurology, Neurosurgery and Psychiatry, 52, 1170-1175.

http://dx.doi.org/10.1136/jnnp.52.10.1170 
Scientific Research Publishing (SCIRP) is one of the largest Open Access journal publishers. It is currently publishing more than 200 open access, online, peer-reviewed journals covering a wide range of academic disciplines. SCIRP serves the worldwide academic communities and contributes to the progress and application of science with its publication.

Other selected journals from SCIRP are listed as below. Submit your manuscript to us via either submit@scirp.org or Online Submission Portal.
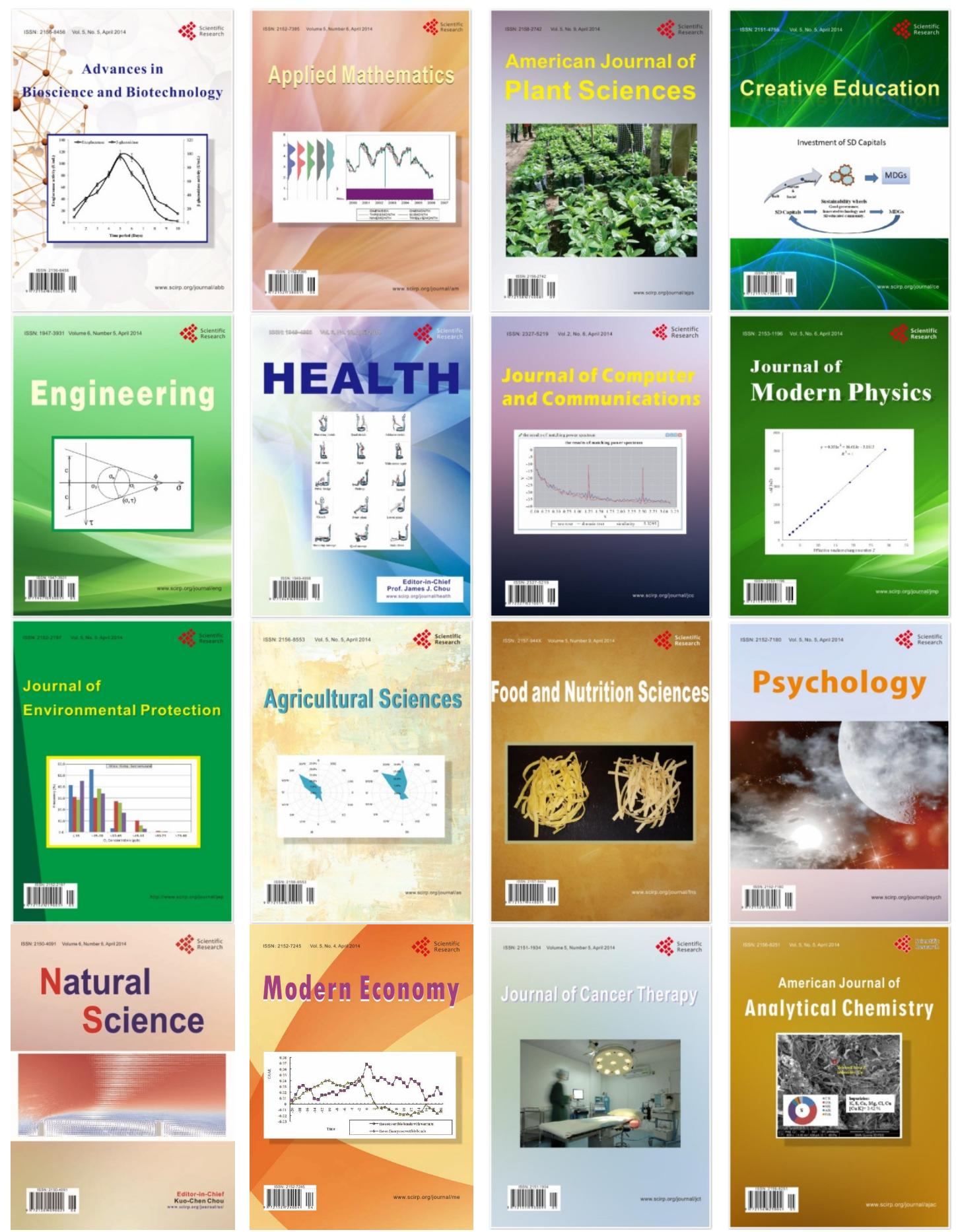